\title{
Applications of 3D Printing in Microscopy Research
}

\section{Kevin Eliceiri}

University of Wisconsin-Madison, Madison, Wisconsin, United States

Although 3D printing is now widely used for medical applications there are lesser-known biomedical applications, including the manufacturing of complex parts for research, such as microfluidic components, and for education. Aiding both research and education, 3D printing can be used to create physical models from biomedical imaging data. Physical 3D models are useful as they allow researchers to hold and feel a structure that they might otherwise only be able to see on a computer screen. These models can prove especially useful in fields where concepts are often difficult to spatially comprehend from a two-dimension (2D) image. For these reasons, these models are also extremely effective teaching tools to help students understand complex 3D biological phenomena, such as membrane architecture and dynamics.

Most 3D printing of biomedical images to date has utilized clinical imaging methods such as computed tomography (CT), positron emission tomography (PET), magnetic resonance imaging (MRI) and 3D ultrasound. Here we describe 3D printing from microscopy methods, such as electron microscopy confocal microscopy and multiphoton microscopy. We describe the workflow, input data and software tools used.

We also discuss other applications of 3D printing in microscopy research including fabricating specialized parts for microscopes, and making imaging phantoms. As well we discus emerging image inspired fabrication approaches including approaches that use microscope based fabrication of tissue patterns from input images. We discuss the state of art of biological tissue printers that can use microscopy images as templates.

As well we review new work in making better more precise parts for microscopy instrumentation include new work in metal printing, We are developing a proof of concept for a multi-source 3D metal printer that utilizes a translating high power array of scanning electron beams. This multi-source electron beam scanner inspired by CT imaging theory requires engineering and design concepts from multiple disciplines to be brought together into a single system. We discuss the current status of this system and the advantages of this technology for specialized part fabrication. 